\title{
Thin silicon films ranging from amorphous to nanocrystalline obtained by Hot-Wire CVD
}

\author{
D. Soler*, M. Fonrodona, C. Voz, J. Bertomeu and J. Andreu \\ Departament de Física Aplicada i Òptica, Universitat de Barcelona, \\ Avda. Diagonal 647, 08028 Barcelona, Spain.
}

\begin{abstract}
In this paper we present results on silicon thin films deposited by Hot-Wire CVD at low substrate temperature $\left(200^{\circ} \mathrm{C}\right)$. Films ranging from amorphous to nanocrystalline were obtained by varying the filament temperature from 1500 to $1800^{\circ} \mathrm{C}$. A crystalline fraction of $50 \%$ was obtained for the sample deposited at $1700^{\circ} \mathrm{C}$. The results obtained seemed to indicate that atomic hydrogen plays a leading role in the obtention of nanocrystalline silicon. The optoelectronic properties of the amorphous material obtained in these conditions are slightly poorer than the ones observed in device-grade films grown by Plasma-Enhanced CVD due to a higher hydrogen incorporation $(13 \%)$.
\end{abstract}

Keywords: Amorphous silicon, nanocrystalline silicon, HWCVD.

\section{Introduction}

Hot-wire CVD (HWCVD) has shown to be a promising technique for the deposition of silicon thin films for photovoltaics and large area electronic applications [1]. This technique allows the deposition of device quality material at high deposition rates with low hydrogen contents and improved stability under light soaking [2]. Although typical depositon temperatures required to obtain device grade materials in HWCVD are higher than the ones required in Plasma-Enhanced CVD (PECVD), several studies have been done in order to lower the deposition temperature so that low cost substrates such as glass, plastic or even polymer foils can be used.

Thin silicon films ranging from amorphous (a-Si:H) to nanocrystalline (nc-Si:H) can be obtained by HWCVD by properly tuning the deposition conditions. This transition has been achieved by varying different technological parameters such as hydrogen dilution [3], substrate temperature [2] and process pressure. In this work we focus on the transition under the variation of the filament temperature $\left(\mathrm{T}_{\mathrm{f}}\right)$. Structural, optical and electrical properties of several samples deposited at low substrate temperature and different $T_{f}$ are presented.

\footnotetext{
* Corresponding author: tel: +34 93 4021134, fax: +34 93 4021138, e-mail: dsoler@ fao.ub.es
} 


\section{Experimental}

Thin silicon films (0.7-0.8 $\mu \mathrm{m}$ thick) were deposited onto Corning 1737 glass and $\mathrm{Si}(111)$ in a HWCVD reactor described elsewhere[4]. The dissociation of the gases was achieved by means of a tantalum filament heated at temperatures in the range of 1500 to $1800^{\circ} \mathrm{C}$. A process pressure of $3.8 \cdot 10^{-2} \mathrm{mbar}$, a substrate temperature of $200^{\circ} \mathrm{C}$ and a pure silane flow of $4 \mathrm{sccm}$ were used.

Crystalline fraction $\left(\mathrm{X}_{\mathrm{c}}\right)$ of the films was deduced from Raman Spectroscopy, following [5]. Hydrogen content and the microstructure factor $\left(\mathrm{R}^{*}\right)[6]$ were obtained from Fourier Transform Infrared (FTIR) spectrometry. Photothermal Deflection (PDS) and Optical Transmition Spectroscopies were used to obtain the band gap $\left(E_{g}\right)$ and the Urbach energy $\left(E_{u}\right)$. Dark conductivity vs. temperature and Steady State Photoconductivity (SSPC) measurements were performed in order to obtain the activation energy $\left(\mathrm{E}_{\mathrm{a}}\right)$, mobility-lifetime product $(\mu \tau)$ and dark conductivity at $33^{\circ} \mathrm{C}\left(\sigma_{\mathrm{d}}\right)$.

\section{Results and discussion}

\subsection{Structural properties}

The transition from a-Si:H to nc-Si:H has been observed under appropiate tuning of the filament temperature. All the samples exhibited a deposition rate of approximately $4 \AA$ $⿱ \mathrm{~s} / \mathrm{s}$, indicating a complete dissociation of the gases at the filament surface regardless the $T_{f}$ in the studied range.

The structural properties of the samples $\left(X_{c}\right.$ and $\left.R^{*}\right)$ as a function of $T_{f}$ can be seen in Fig. 1(a). Samples obtained at $\mathrm{T}_{\mathrm{f}} \geq 1750^{\circ} \mathrm{C}$ showed a clear nanocrystalline character with $\mathrm{X}_{\mathrm{c}}$ greater than $90 \%$. On the other hand, the samples grown at $\mathrm{T}_{\mathrm{f}} \leq 1650^{\circ} \mathrm{C}$ showed amorphous behaviour. For $\mathrm{T}_{\mathrm{f}}$ around $1700^{\circ} \mathrm{C}, \mathrm{X}_{\mathrm{c}}$ in the range of $50 \%$ were obtained, which showed an intermediate regime between both structures. The change in crystallinity seems to be induced by an increase of atomic hydrogen produced at the surface of the wire at higher $T_{f}$.

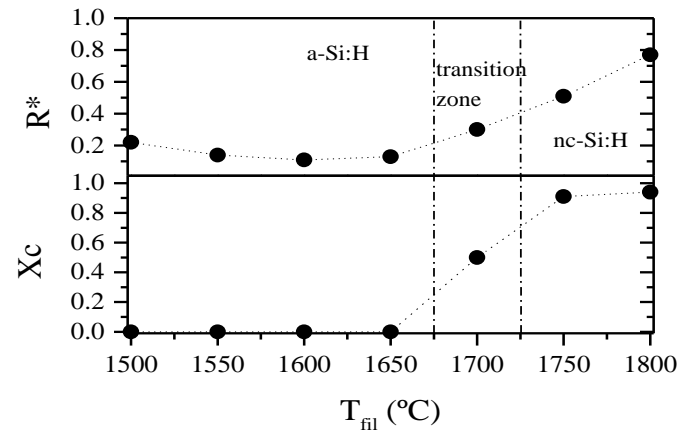

(a)

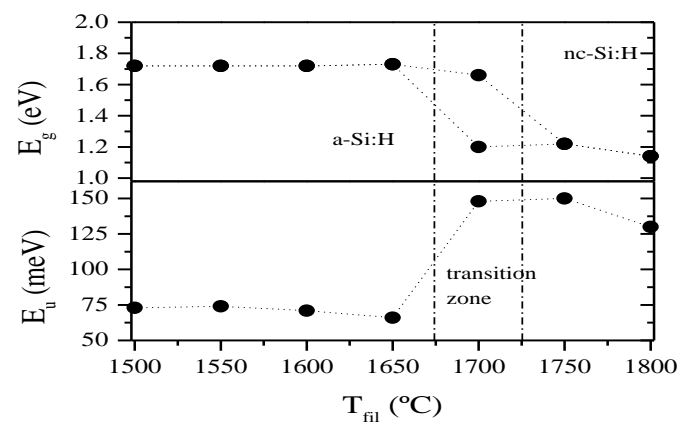

(b)

Fig. 1 Structural properties (a) and optical properties (b) for samples deposited at different filament temperatures. In Fig. 1(b) the nanocrystalline and amorphous gap energies are presented for the sample in the transition zone (see text). 
$\mathrm{R}^{*}$ values in the order of 0.1 have been obtained for the amorphous samples. This reflected the high density of the material. Nanocrystalline samples presented values of $\mathrm{R}^{*}$ higher than 0.4 , increasing with higher $\mathrm{T}_{\mathrm{f}}$.

\subsection{Optical properties}

The $E_{g}$ and $E_{u}$ are presented in Fig. 1(b). Both energies exhibited the same behaviour for $T_{f}$ below $1650{ }^{\circ} \mathrm{C}$, showing a clear amorphous character with an average $\mathrm{E}_{\mathrm{g}}$ of around $1.7 \mathrm{eV}$ and an $\mathrm{E}_{\mathrm{u}}$ of 70 $\mathrm{meV}$. This last value is higher than that expected for device quality material [6]. This fact can be related to the high hydrogen concentration in the samples, which is in the range of $12-13 \%$ for the amorphous samples and around $7 \%$ for the nanocrystalline ones. A reduction of the hydrogen content in the samples requires changes in the technological parameters such as an increase in the substrate temperature [2] or hydrogen dilution [3]. At temperatures above $1750^{\circ} \mathrm{C}$, the optical properties exhibited a nanocrystalline behaviour of the samples, in agreement with the structural properties. In the transition zone, both amorphous and nanocrystalline absorption edges were observed because the material consisted of a mixture of both phases, each being dominant in a different energy range. Fig 1(b) shows the gap energies of both absorption edges for the transition sample.

\subsection{Electrical properties}

Dark conductivity at $33^{\circ} \mathrm{C}, \mathrm{E}_{\mathrm{a}}$ and $\mu \tau$ as a function of $\mathrm{T}_{\mathrm{f}}$ are shown in Fig. 2 . $\mathrm{E}_{\mathrm{a}}$ was calculated by fitting the Arrhenius plot in the range of high temperatures $\left(150-80^{\circ} \mathrm{C}\right)$, where the conduction through extended states is dominant.

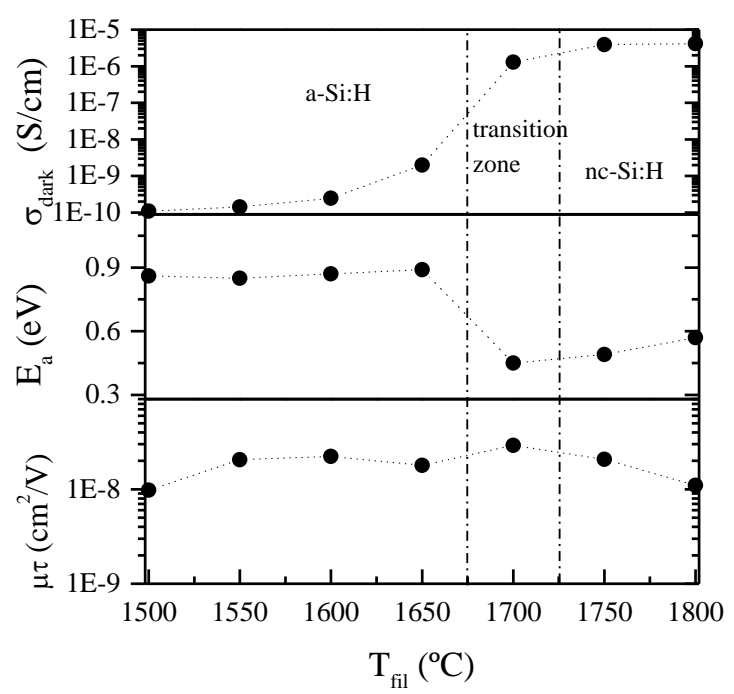

Fig. 2 Electrical properties as a function of the filament temperature

At temperatures lower than $80^{\circ} \mathrm{C}$, a bending in the Arrhenius plot of $\sigma_{\mathrm{d}}$ of the amorphous samples appeared. The exponential behaviour in this range of temperatures corresponds to a lower $\mathrm{E}_{\mathrm{a}}$ and a prefactor $\sigma_{0} \approx 1 \mathrm{~S} / \mathrm{cm}$ which has been attributed to thermal hopping in band tail states [7]. The values 
of $\sigma_{\mathrm{d}}$ shown in Fig. 2 are reasonable for device-quality a-Si:H, in spite of a slight increase in $\sigma_{\mathrm{d}}$ for the sample deposited at $1650^{\circ} \mathrm{C}$. The $\mu \tau$ remains almost constant for all the amorphous samples although its value is slightly lower than the typical ones reported in the literature for device-quality a-Si:H [6]. When we take into account all these facts, it seems to be more difficult to obtain devicegrade material at low substrate temperatures in HWCVD than it is in PECVD.

In the transition zone, the electrical properties of the material seem to be dominated by the nanocrystalline contribution even though a $\mathrm{X}_{\mathrm{c}}$ of $50 \%$ is obtained from the Raman spectra.

Nanocrystalline samples showed a mid-gap character with a slight increase in $E_{a}$ and decrease in $\mu \tau$ when increasing the filament temperature. This could be related to the incorporation of a deep defect level related to impurities coming from the wire as has been observed in recent studies [8].

\section{Conclusion}

Changes in the wire temperature made it possible to control the crystalline fraction of the samples while keeping the deposition rate constant.

Amorphous silicon obtained at low substrate temperature $\left(200^{\circ} \mathrm{C}\right)$ by $\mathrm{HWCVD}$ has a higher hydrogen content than standard PECVD. This fact causes broader tail bands. As a consequence, a higher Urbach energy of the optical absorption edge and a bending in the Arrhenius plot are observed.

Nanocrystalline silicon samples with a high crystalline fraction were obtained without hydrogen dilution of the silane feed. The increasing wire temperature produced a decrease of the mobilitylifetime product which is attributed to slight metallic contamination coming from the wire.

\section{Acknowledgments}

This work was supported by the CICYT of the Spanish Government under the programme TIC 980381-C02-01.

\section{References}

[1] H. Matsumura, J. Appl. Phys. 65 (1989) 4397

[2] A.H. Mahan, J. Carapella, B.P. Nelson, R.S. Crandall, I. Balberg, J. Appl. Phys. 69 (9) (1991) 6728.

[3] S. Bauer, W. Herbst, B. Schröder, H. Oechsner, $26^{\text {th }}$ PVSC IEEE (1997) 719

[4] C. Voz, D. Peiró, J. Bertomeu, D. Soler, M. Fonrodona, J. Andreu, Mater. Sci. Eng. 69-70 (2000) 278.

[5] Z. Iqbal, S. Veprek, A.P. Webb, P. Capezzuto, Solid State Commun. 37 (1981) 993

[6] R.E.I. Schropp, M. Zeman, in: Kluwer Academic Publishers (Eds.), Amorphous and microcrystalline solar cells, 1998

[7] N.F. Mott, E.A. Davis, in: Oxford University Press (Eds.), Electronic processes in noncrystalline materials, (1971) 45

[8] C. Voz, M. Fonrodona, D. Soler, D. Peiró, J.M. Asensi, J. Bertomeu, J. Andreu, Proc. $16^{\text {th }}$

European Photovoltaic Solar Energy Conference, H.S. Stephens \& Associates (UK, 2000) 